\title{
Hopf-algebraic structure of combinatorial objects and differential operators
}

\author{
Robert Grossman* \\ University of Illinois at Chicago \\ Richard G. Larson ${ }^{\dagger}$ \\ University of Illinois at Chicago
}

July, 1989

\begin{abstract}
In this paper we describe a Hopf-algebraic structure on a vector space which has as basis a family of trees, and survey some applications of this structure to combinatorics in Section 1, and to differential operators in Section 2. In Section 3 we indicate some possible future directions for this work.

Much of the material discussed in this paper is work-in-progress. We describe many questions and give some answers.
\end{abstract}

\section{Hopf-algebraic structure of trees and com- binatorics}

We will describe a Hopf-algebraic structure imposed on the vector space spanned by the set of equivalence classes of finite rooted trees, and indicate how this structure can be used to give proofs of classical combinatorial theorems, and how it can be applied in the calculus of differential operators. The

"Supported in part by NASA Grant NAG 2-513.

${ }^{\dagger}$ Supported in part by NSF Grant DMS 870-1085. 
coalgebra structure we define on this space is very similar to the coalgebra structure defined in [9]. However, the coalgebra structure defined there was defined for individual combinatorial objects, rather than for a class of objects such as the family of rooted trees. Other applications of Hopf algebras to combinatorics can be found in [12].

Throughout this paper, $k$ will denote a field of characteristic 0 such as the real numbers or the complex numbers.

By a tree we will mean a finite rooted tree. Let $\mathcal{T}$ be the set of equivalence classes of finite rooted trees, and let $k\{\mathcal{T}\}$ be the $k$-vector space which has $\mathcal{T}$ as a basis.

We first define the coalgebra structure on $k\{\mathcal{T}\}$. If $t \in \mathcal{T}$ is a tree whose root has children $s_{1}, \ldots s_{r}$, the coproduct $\Delta(t)$ is the sum of the $2^{r}$ terms $t_{1} \otimes t_{2}$, where the children of the root of $t_{1}$ and the children of the root of $t_{2}$ range over all $2^{r}$ possible partitions of the children of the root of $t$ into two subsets. The map $\epsilon$ which sends the trivial tree to 1 and every other tree to 0 is a counit for this coproduct. It is immediate that comultiplication is cocommutative.

We next define the algebra structure on $k\{\mathcal{T}\}$. Suppose that $t_{1}, t_{2} \in \mathcal{T}$ are trees. Let $s_{1}, \ldots, s_{\tau}$ be the children of the root of $t_{1}$. If $t_{2}$ has $n+1$ nodes (counting the root), there are $(n+1)^{r}$ ways to attach the $r$ subtrees of $t_{1}$ which have $s_{1}, \ldots, s_{r}$ as roots to the tree $t_{2}$ by making each $s_{i}$ the child of some node of $t_{2}$. The product $t_{1} t_{2}$ is defined to be the sum of these $(n+1)^{r}$ trees. It can be shown that this product is associative, and that the trivial tree consisting only of the root is a right and left unit for this product. It can also be shown that the maps defining the coalgebra structure are algebra homomorphisms, so that $k\{\mathcal{T}\}$ is a bialgebra. For details, sce [3].

The bialgebra $k\{\mathcal{T}\}$ is graded: $k\{\mathcal{T}\}_{n}$ has as basis all trees with $n+1$ nodes. Because the bialgebra $k\{\mathcal{T}\}$ is graded connected, it is a Ilopf algebra. We summarize the above discussion in the following theorem.

Theorem 1 The vector space $k\{\mathcal{T}\}$ with basis all equivalence classes of finite rooted trees is a cocommutative graded connected Hopf algebra.

An important feature of the structure of cocommutative Ilopf algebras over a field of characteristic 0 is the space of primitive elements. If $A$ is a Hopf algebra, then the primitive elements of $A$ are defined by

$$
P(A)=\{a \in A \mid \Delta(a)=1 \otimes a+a \otimes 1\} .
$$


It can be shown that $P(A)$ is a Lie subalgebra of $A^{-}$.

Denote the universal enveloping algebra of the Lie algebra $L$ by $U(L)$.

Two important theorems concerning the structure of cocommutative graded connected Hopf algebras are the following.

Theorem 2 (Milnor-Moore) Let $A$ be a cocommutative graded connected Hopf algebra. Then

$$
A \cong U(P(A))
$$

as Hopf algebras.

Theorem 3 (Poincaré-Birkhoff-Witt) Let $L$ be a Lie algebra with ordered basis $x_{1}, \ldots, x_{n}, \ldots$ Then

$$
\left\{x_{i_{1}}^{e_{1}} \cdots x_{i_{t}}^{e_{t}} \mid i_{1}<\cdots<i_{t} ; 0<e_{k}\right\}
$$

is a basis for $U(L)$.

See [10, page 244] or [14, page 274] for a proof of Theorem 2; see [8, page 159] for a proof of Theorem 3.

A natural question is to find a basis for $P(k\{\mathcal{T}\})$. The answer is given by the following proposition.

Proposition 4 The set of all trees whose root has exactly one child is a basis for $P(k\{\mathcal{T}\})$.

The proof of this proposition is straightforward.

Observing that there is a bijection between trees whose root has one child, and trees with one fewer node, gotten by deleting the root. we get the following immediate consequence.

\section{Corollary 5}

$$
\operatorname{dim} P(k\{\mathcal{T}\})_{n}=\operatorname{dim} k\{\mathcal{T}\}_{n-1}
$$

Putting together Theorems 1, 2, 3, and Corollary 5, and expressing the resulting recurrence relation on $\operatorname{dim} k\{\mathcal{T}\}_{n}$ in terms of generating functions, we get the following classical theorem on the number of rooted trees. 
Theorem 6 (Cayley 1857) Let $a_{n}$ be the number of rooted trees with $n$ nodes. Then

$$
\sum_{n=1}^{\infty} a_{n} z^{n}=z \prod_{n=1}^{\infty} \frac{1}{\left(1-z^{n}\right)^{a_{n}}} .
$$

Other kinds of families of trees give other Hopf algebras. For example, let $\mathcal{O T}$ be the family of equivalence classes of finite rooted ordered trees. (By an ordered tree we mean one in which the children of each node are linearly ordered.) Let $k\{\mathcal{O T}\}$ be the $k$-vector space with basis $\mathcal{O} \mathcal{T}$. Then $k\{\mathcal{O} \mathcal{T}\}$ is a cocommutative Hopf algebra, and

$$
k\{\mathcal{O T}\} \cong k<\mathcal{O} \mathcal{T}_{1}>
$$

the free associative algebra generated by $\mathcal{O} \mathcal{T}_{1}$, the set of ordered trees whose root has exactly one child. Using the structure of this Hopf algebra, we get a Hopf-algebraic proof of a formula for the number of ordered rooted trees discovered by Catalan [2] in 1838 .

\section{Hopf-algebraic structure of trees and dif- ferential operators}

In this section we discuss an extension of the notion of tree, and its application to the study of differential operators.

Let $\left\{E_{1}, \ldots, E_{M}\right\}$ be a finite set of formal symbols. A labeled rooted tre is a finite rooted tree such that each node other than the root is labeled using an element from $\left\{E_{1}, \ldots, E_{M}\right\}$. Note that a label may be used more than once. We denote the set of all labeled rooted trees labeled with $\left\{E_{1}, \ldots\right.$, $\left.E_{M}\right\}$ by $\mathcal{L} \mathcal{T}\left(E_{1}, \ldots, E_{M}\right)$, and the $k$-vector space with this set as basis by $k\left\{\mathcal{L} \mathcal{T}\left(E_{1}, \ldots, E_{M}\right)\right\}$. As in Section 1, we can make $k\left\{\mathcal{L} \mathcal{T}\left(E_{1}, \ldots, E_{M}\right)\right\}$ into a cocommutative graded connected Hopf algebra.

Now consider the situation where the $E_{i}$ are not formal symbols, but are differential operators

$$
E_{i}=\sum_{j=1}^{N} a_{i}^{j}(x) \frac{\partial}{\partial x_{j}},
$$

with $a_{i}^{j}(x) \in R$. The algebra $R$ is typically either the the algebra of polynomial functions $k\left[x_{1}, \ldots, x_{N}\right]$, the algebra of rational functions $k\left(x_{1}, \ldots\right.$, 
$\left.x_{N}\right)$, or the algebra of smooth functions $C^{\infty}\left(k^{N}, k\right)$. We define a map

$$
\psi: k\left\{\mathcal{L} \mathcal{T}\left(E_{1}, \ldots, E_{M}\right)\right\} \rightarrow \text { End } R
$$

as follows. Let $t \in \mathcal{L} \mathcal{T}\left(E_{1}, \ldots, E_{M}\right)$ have $k+1$ nodes, and let $f \in R$. Number the nonroot nodes of $t$ from 1 to $k$. We will make use of the summation indices $i_{1}, \ldots, i_{k}$ in defining $\psi(t) f$. For the root, form the term

$$
T_{0}=\frac{\partial^{r} f}{\partial x_{i_{l}} \cdots \partial x_{i_{l^{\prime}}}}
$$

where the $r$ children of the root are numbered $l, \ldots, l^{\prime}$. For a nonroot node numbered $j$ and labeled with $E_{t}$, form the term

$$
T_{k}=\frac{\partial^{r} a_{t}^{i_{j}}(x)}{\partial x_{i_{l}} \cdots \partial x_{i_{\iota^{\prime}}}},
$$

where the $r$ children of this node are numbered $l, \ldots, l^{\prime}$. Then

$$
\psi(t) f=\sum_{i_{1}, \ldots, i_{k}=1}^{N} T_{k} T_{k-1} \cdots T_{1} T_{0} .
$$

It can be shown that this defines an action of the Hopf algebra $k\left\{\mathcal{L} T\left(E_{1}\right.\right.$, $\left.\ldots, E_{M}\right)$ \} on $R$ which is a measuring.

We now give some examples of applications of this construction.

Applications to symbolic algebra. An important type of computation which is done in the study of differential operators is the computation of the map

$$
k<E_{1}, \ldots, E_{M}>\rightarrow \text { End } R,
$$

where $k<E_{1}, \ldots, E_{M}>$ denotes the free associative algebra generated by the differential operators $E_{1}, \ldots, E_{M}$. The naive computation often leads to cancellation in the computation. For example

$$
\begin{aligned}
&\left(E_{1} E_{2}-E_{2} E_{1}\right) f= \sum_{i_{1}, i_{2}=1}^{N} a_{1}^{i_{1}} \frac{\partial a_{2}^{i_{2}}}{\partial x_{i_{1}}} \frac{\partial f}{\partial x_{i_{2}}}+a_{1}^{i_{1}} a_{2}^{i_{2}} \frac{\partial^{2} f}{\partial x_{i_{1}} \partial x_{i_{2}}} \\
&-a_{2}^{i_{1}} \frac{\partial a_{1}^{i_{2}}}{\partial x_{i_{1}}} \frac{\partial f}{\partial x_{i_{2}}}-a_{2}^{i_{1}} a_{1}^{i_{2}} \frac{\partial^{2} f}{\partial x_{i_{1}} \partial x_{i_{2}}} \\
&=\sum_{i_{1}, i_{2}=1}^{N} a_{1}^{i_{1}} \frac{\partial a_{2}^{i_{2}}}{\partial x_{i_{1}}} \frac{\partial f}{\partial x_{i_{2}}}-a_{2}^{i_{1}} \frac{\partial a_{1}^{i_{2}}}{\partial x_{i_{1}}} \frac{\partial f}{\partial x_{i_{2}}}
\end{aligned}
$$


Now consider the map $k<E_{1}, \ldots, E_{M}>\rightarrow k\left\{\mathcal{L T}\left(E_{1}, \ldots, E_{M}\right)\right\}$ which is defined by sending $E_{i}$ to the tree with two nodes, in which the nonroot node is labeled with $E_{i}$. Then $E_{1} E_{2}-E_{2} E_{1}$ maps to the difference of two trees, in both of which the root has one child, labeled with either $E_{2}$ or $E_{1}$. The child of the root in turn has one child, labeled with either $E_{1}$ or $E_{2}$. In other words, the cancellation which occured in Equation (1) already occurs at the tree level. We have a commutative diagram

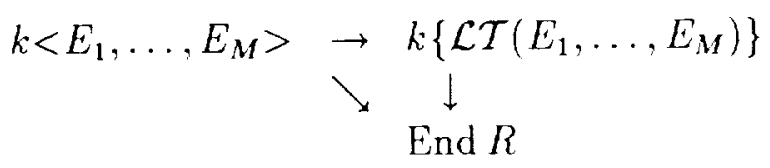

in which it is much more efficient to compute the composition of the rightpointing arrow and the downward-pointing arrow, than it is to compute the diagonal arrow directly. In some common cases, the improvement in efficiency is exponential. A more detailed exposition of this material can be found in [4], [7] and [6].

Solution of nonlinear systems of equations. Another application is the local approximation of a differential equation by an equation which has a symbolic solution. Suppose that

$$
F_{i}=\sum_{j=1}^{N} b_{i}^{j}(x) \frac{\partial}{\partial x_{j}}
$$

where the $b_{i}^{j}(x)$ are not necessarily polynomial functions. Let

$$
F(x, u)=\sum_{i=1}^{M} u_{i}(t) F_{i},
$$

where $t \rightarrow u_{i}(t)$ are fixed time varying functions. We are interested in the differential equation $y^{\prime}(t)=F(y(t), u(t))$. In applications, the $u_{i}$ typically represent controls or other physical parameters describing the system.

It is rare that trajectories of the differential equation can be integrated explicitly in closed form. There is however a simple case in which this is true. Assume that for each $i=1, \ldots, M$, the functions $b_{i}^{j}(x)$ are polynomial functions of the variables $x_{1}, \ldots, x_{j-1}$. In this case trajectories of the differential equation can be written as explict functions involving quadrature of 
the functions $u_{i}$. Vector fields of this form arise when the $F_{i}$ are homogenous of degree 1 with respect to a grading of the underlying vector space $k^{N}$.

Therefore we want to find vector fields

$$
E_{i}=\sum_{j=1}^{N} a_{i}^{j}(x) \frac{\partial}{\partial x_{j}},
$$

where the $E_{i}$ are vector fields with polynomial coefficients homogenous of degree 1 which approximate the $F_{i}$. Specifically, we want to find $E_{i}$ such that

$$
\left.E_{i_{1}} \cdots E_{i_{k}} x^{\beta}\right|_{x=0}=\left.F_{i_{1}} \cdots F_{i_{k}} x^{\beta}\right|_{x=0}
$$

for all $k \leq r$, for some fixed $r$. (IIere $x^{\beta}$ denotes the monomial $x_{1}^{\beta_{1}} \cdots x_{N}^{\beta_{N}}$.) Note that the right-hand sides of Equations (2) are constants; the left-hand sides are nonlinear polynomials in the coefficients of the polynomials $a_{i}^{j}(x)$. By expressing the action of the $E_{i_{1}} \cdots E_{i_{k}}$ using trees, we produce a sequence of systems of nonlinear equations, such that eacil system involves more of the coefficients of the polynomials $a_{i}^{j}(x)$, and such that if we solve one of the systems and substitute its solutions into the next system in the sequence, we get a linear system which we can either solve, or prove insoluble.

For a more detailed discussion of this material, see [5]

Runge-Kutta methods for solving differential equations. An important area in numerical analysis is the study of higher order algorithms for the solution of initial value problems for ordinary differential equations. Specifically, suppose we are solving the differential equation $y^{\prime}=F(y)$, subject to the initial condition $y_{0}=y(0)$. Let

$$
\begin{aligned}
Y_{1} & =y_{0} \\
Y_{2} & =y_{0}+h c_{2,1} F\left(Y_{1}\right) \\
Y_{3} & =y_{0}+h c_{3,1} F\left(Y_{1}\right)+h c_{3,2} F\left(Y_{2}\right) \\
& \vdots \\
Y_{r} & =y_{0}+h c_{r, 1} F\left(Y_{1}\right)+h c_{r, 2} F\left(Y_{2}\right)+\cdots+h c_{r, r-1} F\left(Y_{r-1}^{r}\right)
\end{aligned}
$$

and let

$$
y_{1}=y_{0}+\sum_{i=1}^{r} h b_{i} F\left(Y_{i}\right)
$$


The problem is to find $c_{i, j}$ and $b_{i}$ to give the best approximation $y_{1} \approx y(h)$. Here we can use trees to get equations which the $c_{i, j}$ and $b_{i}$ must satisfy to get an approximation of sufficiently high degree.

This application of trees has been studied extensively by Butcher in [1]. The approach taken there is somewhat different than ours: the existence of a canonical basis for $k\{\mathcal{T}\}$ allows an identification of $k\{\mathcal{T}\}^{*}$ with a completion of $k\{\mathcal{T}\}$, which Butcher makes use of.

In all of these examples there is a common theme: trees allow simple and systematic management of calculations involving higher derivations, a fact which was known to Cayley in the middle of the nineteenth century. We use the Hopf algebra associated with a family of trees systematically to manage calculations involving trces.

\section{$3 \quad$ Future directions}

In this section we discuss some areas for possible future rescarch using the Hopf-algebraic structure of families of trees.

Since $k\left\{\mathcal{L} \mathcal{T}\left(E_{1}, \ldots, E_{M}\right)\right.$ acts on $R$, we have an $R / k$-bialgebra structure on $R \otimes k\left\{\mathcal{L} \mathcal{T}\left(E_{1}, \ldots, E_{M}\right)\right\}$. This allows us to use the structure of $R / k$ bialgebras (as presented in [11] and [13]) to investigate the action of trees as higher derivations of $R$. The $R / k$-bialgebra structure we have described is very dependent on the specific action on $R$, and on the fact that this action is defined in terms of commuting differential operators $\partial / \partial x_{1}, \ldots, \partial / \partial x_{N}$. It is possible that the use of ordered trees rather than trees will climinate this problem.

There is a close connection between functionals on trees and formal power series. More specifically, the coefficients $a_{n}$ of the Taylor series expansion

$$
f(x)=\sum_{n=0}^{\infty} \frac{a_{n}}{n !} x^{n}
$$

satisfy

$$
\left.\frac{d^{n} f}{d x^{n}}\right|_{x=0}=a_{n} .
$$

In a similar fashion, if $E_{1}, \ldots, E_{M}$ are noncommuting differential operators. we can use the set of values $\left.E_{i_{1}} \cdots E_{i_{k}} f\right|_{x=0}$, or the set of values $\left.\psi(t) f\right|_{x=0}$, 
for $t \in \mathcal{L} \mathcal{T}\left(E_{1}, \ldots, E_{M}\right)$. In this way we see that $k\left\{\mathcal{L} \mathcal{T}\left(E_{1}, \ldots, E_{M}\right)\right\}^{*}$ is a generalization of formal power series.

\section{References}

[1] J. C. Butcher, "The Numerical Analysis of Ordinary Differential Equations," Wiley, New York, 1987.

[2] E. Catalan, Note sur une équation aux différences finies, J. Math. Pures Appl. (1) 3 (1838), 508-516.

[3] R. Grossman and R. G. Larson, Hopf-algebraic structure of familics of trees, J. Algebra, to appear.

[4] R. Grossman and R. G. Larson, Labeled Trees and the Algebra of Differential Operators, in "Algorithms and Graphs," R. Bruce Richter, Ed., Contemporary Mathematics, Vol. 89, Amer. Math. Soc., Providence, $1989,81-87$.

[5] R. Grossman and R. G. Larson, Solving Nonlinear Equations From Higher Order Derivations in Linear Stages, Adv. in Math., to appear.

[6] R. Grossman and R. G. Larson, Symbolic computation of derivations using labeled trees, to appear.

[7] R. Grossman and R. G. Larson, Labeled trees and the efficient computation of derivations, in "Proceedings of the ACM-SIGSAM 1989 International Symposium on Symbolic and Algebraic Computation," ACM, $1989,74-80$.

[8] N. Jacobson, "Lie algebras," Interscience, New York, 1962.

[9] S. A. Joni and G.-C. Rota, Coalgebras and bialgebras in combinatorics, Stud. Appl. Math. 61 (1979), 93-139; reprinted in "Umbral Calculus and Hopf Algebras," Amer. Math. Soc., Providence, 1982, 1-47.

[10] J. W. Milnor and J. C. Moore, On the structure of Hopf algebras, Ann. Math. (2) 81 (1965), 211-264. 
[11] W. D. Nichols, The Kostant structure theorems for K/k-Hopf algebras, J. Algebra 97 (1985), 313-328.

[12] W. Nichols and M. Sweedler, Hopf algebras and combinatorics, in "Umbral Calculus and Hopf Algebras," Amer. Math. Soc., Providence, 1982, 49-84.

[13] W. Nichols and B. Weisfeiler, Differential formal groups of J. F. Ritt, Amer. J. Math. 104 (1982), 943-1003.

[14] M. Sweedler, "Hopf Algebras," W. A. Benjamin, New York, 1969. 